\title{
Motivação intrínseca e extrínseca: diferenças no sexo e na idade
}

\author{
Simone Maria Pansera \\ Universidade Federal do Rio Grande do Sul - Porto Alegre - RS - Brasil \\ Nadia Cristina Valentini \\ Universidade Federal do Rio Grande do Sul - Porto Alegre - RS - Brasil \\ Mariele Santayana de Souza \\ Universidade Federal do Rio Grande do Sul - Porto Alegre - RS - Brasil \\ Adriana Berleze \\ Universidade Federal do Rio Grande do Sul - Porto Alegre - RS - Brasil
}

\begin{abstract}
Resumo
A orientação motivacional é um determinante crítico do nível e da qualidade da aprendizagem. Este estudo investigou os níveis de orientação motivacional e as possíveis diferenças entre idade e sexo em 142 crianças de nove e 10 anos alunos de escolas públicas. A "Scale of Intrisic versus Extrinsic Orientation in Classroom" de Harter (1980) foi o instrumento utilizado para a coleta de dados. A escala foi aplicada individualmente e lidaem voz alta pela pesquisadora para melhor compreensão da criança. Os resultados apontam para: (1) níveis moderados de motivação intrínseca em todas as dimensões investigadas; (2) semelhanças na orientação motivacional entre meninos e meninas; (3) semelhanças na orientação motivacional entre as idades. Os níveis moderados de motivação intrínseca apresentados indicam que as crianças deste estudo manifestam interesse nas tarefas e se envolvem com o processo de aprendizagem.
\end{abstract}

Palavras-chave: Motivação; desenvolvimento infantil; educação.

\section{Intrinsic and extrinsic motivation: differences in gender and age}

\begin{abstract}
The motivational orientation is a critical determinant of the level and quality of learning. This study investigated the levels of motivational orientation and the possible differences between age and gender in 142 children of nine and 10 years of public school students. The "Scale of Intrisic versus Extrinsic Orientation in Classroom" Harter (1980) was the instrument used to collect the data. The scale was applied individually and read out by the researcher to better understand by the child. The results indicate: (1) moderate levels of intrinsic motivation in all dimensions investigated; (2) similarities in the motivational orientation between boys and girls; (3) the similarity between the ages motivational orientation. Moderate levels of intrinsic motivation presented indicate that children of this study show interest in the tasks and engage in the learning process.
\end{abstract}

Keywords: Motivation; child development; education.

\section{Motivación intrínseca y extrínseca: diferencias en el sexo y en la edad}

\section{Resumen}

La orientación motivacional es un determinante crítico del nivel y de la calidad del aprendizaje. Este estudio investigó los niveles de orientación motivacional y las posibles diferencias entre edad y sexo en 142 niños de nueve y 10 años alumnos de escuelas públicas. La "Scale of Intrisic versus Extrinsic Orientation in Classroom" de Harter (1980) fue el instrumento utilizado para la colecta de datos. La escala fue aplicada individualmente y leída en voz alta por la investigadora para mejor comprensión del niño. Los resultados apuntan para: (1) niveles moderados de motivación intrínseca en todas las dimensiones investigadas; (2) semejanzas en la orientación motivacional entre niños y niñas; (3) semejanzas en la orientación motivacional entre las edades. Los niveles moderados de motivación intrínseca presentados indican que los niños de este estudio manifiestan interés en las tareas y se involucran con el proceso de aprendizaje.

Palabras clave: Motivación; desarrollo infantil; educación. 


\section{Introdução}

A motivação é a força que emerge, regula e sustenta as ações de cada indivíduo; ela é um processo complexo que influencia o início de uma atividade e a sua manutenção com persistência e vigor ao longo do tempo (Harter, 1981; Deci, Vallerand, Pelletier, \& Ryan, 1991; Ryan \& Deci, 2000). A motivação não deve ser considerada como um traço relativamente estável do comportamento humano, pois ela muda em função das características do contexto ao qual o indivíduo está exposto (Harter, 1981; Deci \& cols., 1991; Ryan \& Deci, 2000; Berleze, Vieira, \& Krebs, 2002; Parish \& Treasure, 2003; Martinelli \& Bartholomeu, 2007; Lens, Matos,\& Vansteenkiste, 2008; Martinelli \& Genari, 2009; Paiva \& Boruchovitch, 2010). A motivaçãointrínseca é primordial para o desenvolvimento humano e é estudada por psicólogos e educadores em todas as esferas do desenvolvimento humano, especialmente pelo seu papel essencial na aprendizagem escolar (Zisimopoulos \& Galanaki, 2009).

A motivação intrínseca é reconhecida como um mediador importante na conquista da competência e autoconceito do indivíduo sobre o ambiente (Harter, 1978, 1981; White, 1959). A criança motivada intrinsecamente em um determinado domínio do comportamento humano demonstra esforço para demonstrar competência nesse domínio. Uma criança motivada extrinsecamente para uma tarefa pode não empenhar-se para realizar a tarefa e consequentemente apresentar sentimentos mais inferiores de competência (Harter, 1981; Goudas, Dermitzaki, \& Bagiatis, 2000; Guay, Boggiano, \& Vallerand, 2001; Weiss \& Amorose, 2005; Valentini, 2006; Zisimopoulos \& Galanaki, 2009).

A motivação intrínseca se refere à execução de uma determinada atividade pelo prazer que esta pode proporcionar (Goudas \& cols., 2000; Lepper, Corpus, \& Iyengar, 2005; Villwock, 2005; Corpus, McClintic-Gilbert, \& Hayenga, 2009; Martinelli \& Sisto, 2010; Paiva \& Boruchovitch, 2010), sem a necessidade de pressões externas, prêmios ou recompensa; o indivíduo busca a atividade por esta ser interessante, envolvente e geradora de satisfação (Valentini, 2002; Guimarães \&Boruchovitch, 2004; Martinelli \& Bartholomeu, 2007; Neves \& Boruchovitch, 2007; Villwock \& Valentini, 2007; Martinelli \& Genari, 2009; Zisimopoulos \& Galanaki, 2009). Do contrário, a criança com orientação motivacional extrínseca, busca a aprovação do professor e de pares, uma necessidade de promoção (Harter, 1981; Ryan \& Deci, 2000; Valentini, 2002; Guay \& cols., 2001; Guimarães \&Boruchovitch, 2004; Lepper \& cols., 2005; Martinelli \& Bartholomeu, 2007; Martinelli \& Genari, 2009; Martinelli \& Sisto, 2010; Paiva \& Boruchovitch, 2010) ou ainda se envolve com tarefas apenas por acreditar que haverá alguma consequência, positiva ou negativa, sem interesse na aprendizagem (Goudas \& cols., 2000; Guay \& cols., 2001).

Uma criança com orientação motivacional intrínseca está mais impulsionada a estudar, a iniciar uma atividade e permanecer nela até o fim (Deci \& cols., 1991; Neves \& Boruchovitch, 2007; Villwock \& Valentini, 2007; Martinelli \& Genari, 2009; Zisimopoulos \& Galanaki, 2009; Paiva \&Bo- ruchovitch, 2010). Entretanto, é preciso examinar a relativa força da motivação intrínseca e extrínseca e suas relações com a orientação motivacional. Embora pareçam ser contraditórias, existem situações em que o interesse intrínseco e recompensa extrínseca podem colaborar para motivar uma criança à aprendizagem. Assim, uma criança pode ser curiosa e gostar de trabalho desafiador, mas também vê na aprovação do professor um incentivo à aprendizagem, ou ainda, a criança pode preferir trabalhar de forma independente, mas em um determinado momento precisar da ajuda do professor (Harter, 1981; Lepper \& cols., 2005; Martinelli \& Genari, 2009; Zisimopoulos \& Galanaki, 2009; Paiva \& Boruchovitch, 2010).

Harter $(1981,1999)$ propõe um modelo de motivação intrínseca para a maestria em crianças no qual componentes motivacionais (desafios, curiosidade/interesse, maestria independente), bem como componentes cognitivos-informacionais (julgamento e critérios internos) compõem o constructo da motivação; alterações nesses componentes podem levar a adoção de uma orientação mais intrínseca e ou extrínseca dependendo da experiência sendo vivenciada e do desenvolvimento da criança. Tal modelo tem sido adotado no meio educacional pelas possibilidades investigativas da criança em desenvolvimento. No contexto escolar, a motivação é um determinante crítico do nível e da qualidade da aprendizagem e do desempenho, uma vez que estudos têm evidenciado que ao longo do processo de desenvolvimento a motivação intrínseca sofre decréscimo, sendo que crianças ao final das séries iniciais já não apresentam a mesma curiosidade e gosto elevado pelo desafio observados nos primeiros anos da infância (Harter, 1981; Wigfield \& Guthiere, 1997; Lepper \& cols., 2005; Corpus \& cols., 2009; Martinelli \& Genari, 2009; Martinelli \& Sisto, 2010; Paiva \& Boruchovitch, 2010; Gillet, Vallerand, \& Lafrenière, 2011).

A motivação intrínseca é um importante mediador do engajamento e conquistas escolares (Ames, 1992a, 1992b), potencializando o processo de aprendizagem (Zisimopoulos \& Galanaki, 2009; Paiva \&Boruchovitch, 2010). Promover na criança a preferência por desafios, curiosidade e julgamento autônomo em tarefas escolares fortalece a motivação intrínseca para aprender (Harter, 1981; Villwock \& Valentini, 2007). Considerando-se ainda a estrutura cognitivo-informacional da motivação, quando oportunidades são criadas no ambiente escolar para a criança mostrar o que sabe e que parâmetros utiliza para tomar decisões, a motivação intrínseca se fortalece (Harter, 1981, 1992; Ames, 1992a, 1992b).

Embora fundamental para a aprendizagem, pesquisas no meio escolar sobre orientação motivacional são bastante limitadas epouco tem se discutido sobre asdiferenças em relação ao sexo e as repercussões que as mudanças na estrutura escolar podem ocasionar na motivação infantil. Especificamente Harter $(1978,1980)$ sugere que modificações importantes ocorrem a partir do quarto ano de escolarização, momento no qual a criança passa a entender mais plenamente as regras e protocolos da escola e comporta-se extrinsecamente motivada para responder a essas demandas. Outro fator importante é que a partir do quarto ano 
começam a ser criadas expectativas a respeito da nova organização escolar, que causa, muitas vezes, incertezas e inseguranças nos alunos, sendo um período de transição entre a unidocência para a múltipla-docência com diferentes professores em diferentes disciplinas (Prati \& Elzirik, 2006). O próprio sistema escolar é explicito quanto aos níveis de exigência e desafios maiores que crianças terão de enfrentar, consequentemente precisando demandar um esforço muito maior para atingir os objetivos para a aprovação (Prati \& Elzirik, 2006), tornando a quarta série um período em que, explicitamente, o sistema escolar volta-se para a cobrança de demandas fundamentadas em parâmetros extrínsecos.

Dessa forma, o objetivo deste estudo foi investigar a orientação motivacional nas suas diferentes dimensões em escolares de ambos os sexos de 9 e 10 anos de idade. Os seguintes objetivos específicos foram estabelecidos: (1) comparar a orientação motivacional nas diferentes dimensões nos escolares quanto ao sexo; e (2) comparar a orientação motivacional nas diferentes dimensões nos escolares em diferentes idades. As seguintes hipóteses foram adotadas: (1) meninos e meninas não apresentariam diferenças significativas quanto a sua orientação motivacional; e (2) crianças de menor idade apresentariam níveis mais elevados de motivação intrínseca que crianças com idade mais avançada.

\section{Método}

\section{Participantes}

Fizeram parte desta pesquisa descritiva e com delineamento transversal (Thomas \& Nelson, 2002), 142 crianças (75 meninos e 67 meninas) com 9 anos (entre 8,7 a 9,$5 ; M=9,3, D P=0,57$ ) e 10 anos (entre 10,5 a 11,$6 ; M=10,9$, $\mathrm{DP}=0,42$ )de idade, matriculados em escolas da rede pública de ensino do sul do Brasil. As escolas estão localizadas em bairros residenciais e fazem parte da rede pública de ensino com enfoque no ensino infantil e fundamental.As escolas caracterizavam-se por terem salas de aula pequenas para os alunos e com poucos recursos (classes e quadro para o professor escrever, por exemplo). Algumas escolas tinham sala de informática, no entanto era pouco frequentada pelos alunos devido às más condições dos equipamentos ou por não haver equipamentos suficientes para todos os alunos. Em relação ao ambiente externo, destinado às aulas de educação física, havia espaços demarcados para que as crianças jogassem voleibol e futsal, no entanto, em muitos desses locais as pinturas já estavam apagadas, podendo confundir os limites dos espaços; além disso, não havia ginásio que possibilitasse a prática de atividades físicas em dias de chuva. Em algumas das escolas havia um espaço ou uma quadra coberta que se mais de uma turma tivesse aula de educação física no mesmo momento, o que é comum nas escolas, impossibilitaria a prática de atividades com muita movimentação devido ao espaço restrito desses ambientes. Profissionais da área de educação física atuavam em todas as turmas. As escolas estavam inseridas em bairros considerados de classe média e classe média-baixa. A maioria das famílias atendidas por essas escolas tinha como renda mensal até três salários mínimos. Após a seleção das escolas, foram selecionadas as turmas de acordo com as idades previstas. A todos os alunos regularmente matriculados $\mathrm{e}$ dentro da faixa etária estipulada foi entregue o termo de consentimento livre e esclarecido para ser assinado pelos pais ou responsáveis legais e somente participaram da pesquisa os alunos que trouxeram o termo assinado ${ }^{1}$.

\section{Instrumento e coleta de dados}

Para a coleta de dados referente à orientação motivacional foi utilizada a "Scale of Intrisic versus Extrinsic Orientation in Classroom" (Harter, 1980). Essa escala é formada por cinco dimensões (desafio, curiosidade, independência, julgamento e critério), contendo seis itens em cada. As dimensões desafio, curiosidade e independência são definidas como motivacionais, já que respondem a questões como o que a criança quer, gosta e prefere fazer. As dimensões julgamento e critério são definidas como cognitivo-informacionais, pois avaliam o que a criança sabe, como toma as decisões e o quanto ela tem aprendido sobre as regras da escola. Os valores para cada item da escala correspondem de um (motivação extrínseca) a quatro (motivação intrínseca).A pontuação total vai de 30 a 120 pontos.

Essa escala foi validada em seu conteúdo no Brasil (Villwock, 2005; Almeida, Rocha, Souza, \& Villwock, 2007) em 258 crianças brasileiras com idade entre oito e 13 anos, através de cinco etapas: (1) versão preliminar com tradução inversa; (2) aplicação na população; (3) avaliação da clareza e da pertinência; (4) validade concomitante do conteúdo; e (5) fidedignidade e consistência interna (Villwock, 2005), sendo que os resultados indicaram que a versão preliminar atendeu aos critérios de clareza e pertinência esperados. Estudos prévios com crianças brasileiras têm apresentado resultados semelhantes aos do estudo original (Harter 1981). No estudo de Almeida e cols. (2005) o coeficiente de fidedignidade foi significativo e forte $(p=0,82)$ e no estudo de Villwock (2005) o coeficiente total foi de 0,81 . No presente estudo o índice de fidedignidade foi de 0,75.

Durante a aplicação da "Scale of Intrisic versus Extrinsic Orientation in Classroom" (Harter, 1980) os avaliados foram solicitados a decidir com qual das duas crianças descritas elas se pareciam mais e então marcar se a descrição escolhida era realmente verdadeira ou somente em parte verdadeira. Cada questão foi lida para a criança em voz alta para que ela compreendesse melhor e assinalasse a resposta escolhida. Para todos os participantes uma primeira questão foi conduzida como exemplo. A aplicação da escala aconteceu em dois momentos, com teste e reteste para analisar a fidedignidade da escala na amostra do presente estudo.

1 A pesquisa foi aprovada pelo comitê de ética ( $\left.n^{\circ} 2003109\right)$. 


\section{Análise estatística}

O estudo de Harter (1992) serviu de base para a categorização da orientação motivacional em motivação extrínseca, motivação intrínseca moderada ou motivação intrínseca alta. Para categorizar a orientação motivacional das crianças foi calculada a média e desvio padrão dos valores referentes àsoma de cada domínio dos participantes do presente estudo. Após ser determinada a média e desvio padrão, foi utilizada a categorização sugerida por Harter (1992) em que crianças que apresentaram valores referentes a um desvio padrão ou mais acima da média foram categorizadas com motivação intrínseca alta; crianças com valores referentes a entre um desvio padrão abaixo da média e um desvio padrão acima da média foram categorizadas com motivação intrínseca moderada e crianças com valores referentes a um desvio padrão ou mais abaixo da média foram categorizadas com motivação extrínseca.

Para a estatística descritiva foram utilizados média e desvio padrão. Para as comparações nas diferentes faixas etárias e para os sexos foram utilizadas análises de variâncias (ANOVA). O nível de significância $p<0,05$ foi adotado. O pacote estatístico SPSS versão 16.0 para Windows foi utilizado na análise dos dados deste estudo.

\section{Resultados}

Os participantes do presente estudo apresentaram motivação intrínseca moderada para a dimensão desafio
$(67 \%)$, curiosidade $(63 \%)$, independência $(59 \%)$, julgamento (70\%) e critério (69\%), conforme os critérios de categorização propostos por Harter (1992). As médias mais altas foram observadas para as dimensões desafio $(M=2,91, D P=0,63)$; curiosidade $(M=3,11, D P=0,54)$ e independência $(M=2,75$, $\mathrm{DP}=0,58)$. Já para as dimensões julgamento $(M=1,93$, $\mathrm{DP}=0,63)$ e critério $(\mathrm{M}=2,38, \mathrm{DP}=0,77)$ as médias foram mais baixas. A seguir os dados serão apresentados quanto ao sexo e a faixa etária das crianças participantes do estudo. Os dados desta análise são apresentados na Figura 1.

ANOVA OneWay foi aplicado para as comparações entre os sexos na motivação. Os resultados não revelaram diferença estatisticamente significativa na orientação motivacional geral entre os sexos $(F(1,140)=0,09 ; p=0,77)$, bem como para as dimensões desafio $(F(1,140)=0,01$; $p=0,94)$; curiosidade $(F(1,140)=2,19 ; p=0,14)$; independência $(F(1,140)=0,13 ; p=0,72)$; julgamento $(F(1,140)=0,05$; $p=0,82)$; e critério $(F(1,140)=0,06 ; p=0,80)$. ANOVA OneWay foi utilizada para as comparações nas idades. Os resultados não revelaram diferença estatisticamente significativa na orientação motivacional geral nas diferentes idades $(F(1,140)=1,95 ; p=0,16)$. Assim, também nãoforam verificadas diferenças estatisticamente significativas entre as idades para as dimensões: desafio $(F(1,140)=0,73$; $p=0,39)$; curiosidade $(F(1,140)=1,93 ; p=0,17)$; independência $(F(1,140)=2,69 ; p=0,10)$; julgamento $(F(1,140)=0,57$; $p=0,45)$; e critério $(F(1,140)=1,91 ; p=0,17)$. As médias e desvios padrões da motivação por sexo e idade são apresentados na tabela 1.

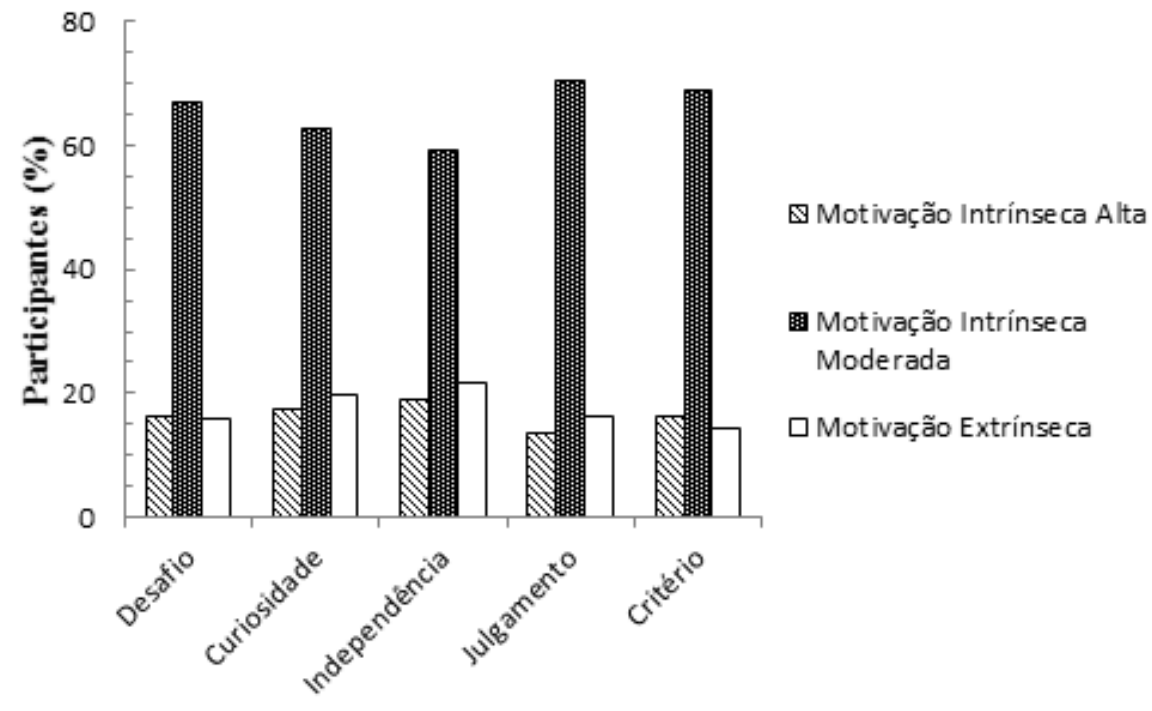

Figura 1. Níveis da orientação motivacional para cada dimensão. 
Tabela 1. Médias e desvios padrão de orientação motivacional nas diferentes dimensões em relação ao sexo e à idade.

\begin{tabular}{llllll}
\hline \multirow{2}{*}{ Dimensões } & $\begin{array}{l}\text { Geral } \\
(\mathbf{M} \pm \mathrm{DP})\end{array}$ & \multicolumn{2}{l}{ Sexo $(\mathrm{M} \pm \mathrm{DP})$} & \multicolumn{2}{l}{ Idades (M $\pm \mathrm{DP})$} \\
\cline { 3 - 6 } & & Masculino & Feminino & $\mathbf{9}$ anos & $\mathbf{1 0}$ anos \\
\hline Desafio & $2,91 \pm 0,63$ & $2,91 \pm 0,65$ & $2,90 \pm 0,61$ & $2,96 \pm 0,65$ & $2,87 \pm 0,62$ \\
\hline Curiosidade & $3,11 \pm 0,54$ & $3,04 \pm 0,57$ & $3,18 \pm 0,50$ & $3,17 \pm 0,53$ & $3,05 \pm 0,55$ \\
\hline Independência & $2,75 \pm 0,58$ & $2,77 \pm 0,55$ & $2,73 \pm 0,61$ & $2,83 \pm 0,56$ & $2,68 \pm 0,58$ \\
\hline Julgamento & $1,93 \pm 0,63$ & $1,94 \pm 0,65$ & $1,91 \pm 0,62$ & $1,88 \pm 0,65$ & $1,96 \pm 0,63$ \\
\hline Critério & $2,38 \pm 0,77$ & $2,36 \pm 0,81$ & $2,39 \pm 0,72$ & $2,47 \pm 0,78$ & $2,30 \pm 0,74$ \\
\hline Média Geral & $2,61 \pm 0,40$ & $2,60 \pm 0,40$ & $2,62 \pm 0,40$ & $2,66 \pm 0,38$ & $2,57 \pm 0,42$ \\
\hline
\end{tabular}

\section{Discussão}

As crianças do presente estudo apresentaram motivação intrínseca moderada na média geral e em todas as dimensões estudadas. Médias mais altas foram observadas para as dimensões desafio, curiosidade e independência. Essas dimensões são consideradas motivacionais para a maestria de tarefas. Foram observadas médias mais baixas para as dimensões julgamento e critério, subescalas consideradas cognitivas informacionais. Esses resultados também foram observados nos estudos conduzidos nos dados originais nos Estados Unidos (Harter, 1981, 1992) e em pesquisa prévia nacional com crianças de 8 a 10 anos (Villwock \& Valentini, 2007). Esses resultados indicam que as crianças deste estudo são curiosas, apreciam desafios e gostam de trabalhar de forma independente, porém, elas ainda dependem do professor para julgar suas habilidades e para construir critérios internos de sucesso ou fracasso (Harter, 1981; Lepper \& cols., 2005; Villwock \& Valentini, 2007). A importância destes resultados se torna mais evidente quando se observa que crianças com orientação motivacional intrínseca têm melhor desempenho em sala de aula (Wigfield \& Guthrie, 1997; Lepper \& cols., 2005; Martinelli \& Genari, 2009; Paiva \&Boruchovitch, 2010), maior satisfação e participação durante a prática esportiva (Fernandes \& Vasconcelos-Raposo, 2005) e mais empenho e persistência para realizar as aulas de educação física (Carratalá, Guzmán, Carratalá, \& García, 2004). Lepper e cols. (2005) observaram que quanto maior a motivação intrínseca menor é a preferência por trabalho fácil no ambiente de aprendizagem.

Os níveis moderados de motivação intrínseca observados no presente estudo podem indicar prazer e envolvimento nas atividades e na aprendizagem e no ambiente escolar (Villwock \& Valentini, 2007). Um fator também importante refere-se à fase de desenvolvimento que essas crianças se encontram, ou seja, essas crianças possivelmente estão em processo de troca de uma motivação mais intrínseca para uma motivação mais extrínseca, na qual agradar aos professores e obter recompensas externas, como aprovação e aceitação social,passam a ter uma maior influência nos comportamentos escolares (Villwock \& Valentini, 2007).

Destaca-se que a motivação intrínseca e a motivação extrínseca coexistem em sala de aula e que o fundamental não é se a criança é intrínseca ou extrinsecamente motivada, mas a frequência de cada comportamento (Lepper \& cols., 2005; Martinelli \& Genari, 2009). A criança pode buscar uma atividade pelo prazer inerente e ao mesmo tempo estar prestando atenção às consequências extrínsecas desta atividade no contexto (Villwock \& Valentini, 2007). Entretanto, deve-se tomar cuidado com a demanda de constantes imposições e restrições extrínsecas durante a aprendizagem na escola. A combinação de aprendizagens descontextualizadas com a constante cobrança por boas notas podem inibir ainda mais o interesse intrínseco e o envolvimento das crianças com os processos de aprendizagem e autonomia para aprender (Ginsburg \& Broestein, 1993; Goudas \& cols., 2000; Guay \& cols., 2001; Lepper \& cols., 2005; Villwock \& Valentini, 2007).

Nas comparações conduzidas entre os sexos, as crianças do presente estudo apresentaram níveis semeIhantes de orientação motivacional na média geral, assim como nas dimensões desafio, curiosidade, independência, julgamento e critério. Esses resultados suportam a primeira hipótese da pesquisa, de que meninos e meninas apresentariam semelhança nos níveis de orientação motivacional. No entanto, os resultados sobre esse tema são contraditórios até o momento. Alguns estudos corroboram com os resultados do presente estudo com similaridade nas idades (Harter, 1981; Villwock \& Valentini, 2007). Porém, outros estudos indicam que as meninas são mais motivadas intrinsecamente de forma geral (Paiva \& Boruchovitch, 2010) em atividades específicas, como leitura, do que os meninos (Wigfield \& Guthrie, 1997). Além disso, os meninos, muitas vezes, acreditam que a inteligência é algo estável e imutável, o que influencia negativamente a motivação intrínseca para a aprendizagem e o sucesso escolar (Paiva \& Boruchovitch, 2010). Os resultados encontrados por nós podem estar 
relacionados às estratégias educacionais que não reforçam diferenças, as quais proporcionam oportunidades semelhantes de construção da orientação motivacional entre meninos e meninas. Entretanto, mais estudos são necessários para a confirmação ou não desse fato.

Quando as comparações foram realizadas nas diferentes idades, as crianças do presente estudo não apresentaram diferenças significativas na orientação motivacional geral, assim como nas dimensões desafio, curiosidade, independência, julgamento e critério, não suportando a segunda hipótese deste estudo. Esses resultados contrariam o modelo proposto por Harter (1981) e diferem também de pesquisas prévias conduzidas em diversos países com crianças em faixas etárias mais amplas (Harter, 1981; Wigfield \& Guthrie, 1997; Lepper \& cols., 2005; Corpus \& cols., 2009; Martinelli \& Genari, 2009; Martinelli \& Sisto, 2010; Paiva \& Boruchovitch, 2010; Gillet \& cols., 2011), nas quais a diminuição da motivação intrínseca conforme o aumento da idade e de anos de escolaridade foi observada.

Os estudos internacionais e nacionais discutidos previamente investigaram faixas etárias maiores do que as do presente estudo. Nos Estados Unidos (Harter, 1981; Lepper \& cols., 2005) e Canadá (Gillet \& cols., 2011) os estudos foram conduzidos com crianças e adolescentes, entre 8-9 até 14-17 anos frequentando da terceira série do ensino fundamental até os últimos anos do ensino médio. Nos estudos conduzidos no Brasil, três no estado de São Paulo, crianças entre 7 e 13 anos cursando da $3^{\circ}$ a $5^{\circ}$ ano do ensino fundamental foram investigadas (Martinelli \& Genari, 2009; Martinelli \& Sisto, 2010: Paiva \& Boruchovitch, 2010). Devido às mudanças cognitivas que ocorrem nesses períodos, os estudos indicaram diferenças entre as idades. No presente estudo com a limitação de duas faixas etárias, e consequentemente duas etapas escolares, não foram encontradas diferenças, embora a expectativa fosse de que as mudanças curriculares poderiam ser fatores causadores de mudanças na orientação motivacional das crianças do quarto ano. Entretanto, um estudo no sul do Brasil reportou também estabilidade de escores para a motivação intrínseca em uma faixa etária menor, dos sete aos nove (Villwock, 2005).

Destacamos ainda que possíveis limitações podem decorrer também do uso do próprio instrumento de Harter (1980), o qual mensura a orientação motivacional somente em extrínseca e intrínseca e não em um continuum motivacional (Deci \& cols., 1991); e, ainda que instrumentos auto-avaliativos podem conduzir crianças a apresentarem respostas que são mais socialmente aceitas, embora essa tendência seja mais prevalente em crianças pré-escolares (Harter, 1978, 1999).

\section{Considerações Finais}

O estudo investigou a orientação motivacional em diferentes dimensões de estudantes de escolas públicas de ambos os sexos e com idade de nove e 10 anos. Observou-se um predomínio de motivação intrínseca moderada em todas as dimensões investigadas, bem como não foram encontradas diferenças entre os sexos e as idades nas diversas dimensões da orientação motivacional.

A não observação de diferenças entre os sexos sugere que até o mormente as estratégias educacionais favorecem a equidade entre os gêneros. Quando estratégias educacionais que não reforcem diferenças são implementadas, há uma maior chance de ambos os sexos terem prazer e interesse em realizar as mais variadas atividades, não estigmatizando, por exemplo, algumas atividades são mais propícias para meninas e outras para meninos.

Apesar da limitação quanto a amplitude de idade no presente estudo, o fenômeno de uma mudança mais efetiva para um orientação extrínseca após quatro anos de escolarização não foi evidenciado no presente estudo, crianças de 9 e 10 anos mostraram-se semelhantes em níveis moderados na orientação motivacional. Talvez este ambiente escolar, por mais limitado que seja em recursos materiais, conforme observado no presente estudo, esteja de alguma forma preservando a autonomia, o julgamento independente e os desafios para que as crianças do quarto ano preservem a motivação mais intrinsecamente orientada e consequentemente engajada nas atividades pela sensação de prazer em realizá-las e não pela dependência de um estímulo externo. Consequentemente, a motivação intrínseca é um importante mediador do envolvimento da criança no processo de aprendizagem. Propiciar uma aprendizagem significativa, em um ambiente que promova a autonomia e a curiosidade, desafio, maestria e julgamento independente, são importantes estímulos para a preservação da motivação intrínseca para a aprendizagem.

\section{Referências}

Ames, C. (1992a). Achievement goals, motivational climate, and motivational processes. Em G. C. Roberts (Org.), Motivation in sport and exercise (pp. 161-176). Champaign: Human Kinetics Publishers.

Ames, C. (1992b). Classroom: goals, structures, and student motivation. Journal of Educational Psychology, 84(3), 409-414.

Almeida, G., Rocha, C. H., Souza, M.C., \& Villwock, G. M. C. (2005). Orientação intrínseca e extrínseca: validação de um instrumento de medida [Resumo]. Em Salão de Iniciação Cientifica, Livro de Resumos, (p.514). Porto Alegre: UFRGS.

Berleze, A., Vieira, L. F. \& Krebs, R. J. (2002). Motivos que levam crianças à prática de atividades motoras na escola. Revista da Educação Física,13(1), 99-107.

Carratalá, E., Guzmán, J. F.,Carratalá, V. \& García, A. (2004).La diversión en la práctica deportiva en función del modelo jerárquico de la motivación: un estudio con deportistas de especialización deportiva. Motricidad. European Journal oh Human Movement, 15 
Corpus, J. H., McClintic-Gilbert, M. S., \& Hayenga, A. O. (2009). Within-year changes in children's intrinsic and extrinsic motivational orientations: contextual predictors and academic outcomes. Contemporary Educational Psychology,34 (2), 154-166.

Deci, E. L., Vallerand, R. J., Pelletier, L. G., \& Ryan, R. M. (1991). Motivations and education: the self-determination perspective. Educational Psychologist, 26 (3,4), 325-346.

Fernandes, H. M. \& Vasconcelos-Raposo, J. (2005). Continuum de auto-determinação: validade para a sua aplicação no contexto desportivo. Estudos de Psicologia, 10(3), 385-395.

Gillet, N., Vallerand, R. J. \& Lafrenièrem, A. K. (2011). Intrinsic and extrinsic school motivation as a function of age: the mediating role of autonomy support. Social Psychology of Education, 14.

Ginsburg, G. S. \& Broenstein, P. (1993). Family factors related children's intrinsic/extrinsic motivational orientation and academic performance. Child Development, 14 (5), 1461-1474.

Goudas, M., Dermitzaki, I., \& Bagiatis, K. (2000).Predictors of students intrinsic motivation in school physical education. Journal of Psychology of Education, 15(3), 271-280.

Guay, F., Boggiano, A. K., \& Vallerand, R.J. (2001). Autonomy support, intrinsic motivation and perceived competence: conceptual and empirical linkages. Personality and Social Psychology Bulletin, 27 (6), 643-650

Guimarães, S. E. R. \& Boruchovitch, E. (2004). O estilo motivacional do professor e a motivação intrínseca dos estudantes: uma perspectiva da teoria da autodeterminação. Psicologia: Reflexão e Crítica, 17(2), 143-150.

Harter, S. (1978). Effectance motivation reconsidered: toward a developmental model. Human Development, 21 (1), 24-64.

Harter, S. (1980). A scale of intrinsic versus extrinsic orientation in the classroom. Colorado: University of Denver.

Harter, S. (1981). A new self-report scale of intrinsic versus extrinsic orientation in the classroom: motivational and informational components. Developmental Psychology, 17 (3), 300-312.

Harter, S. (1992). The relationship between perceived competence, affect, and motivational orientation within the classroom: processes and patterns of changes. Em A. K. Boggiano, \& T. S. Pittman (Orgs.), Achievement and Motivation: a social-development perspective. Cambridge: Cambridge University press.

Harter, S. (1999).The Construction of the Self: a developmental perspective. New York: The Guildford Press.

Lens, W., Matos, L., \& Vansteenkiste, M. (2008). Professores como fonte de motivação dos alunos: o quê e o porquê da aprendizagem do aluno. Educação, 31 (1), 17-20.
Lepper, M. R., Corpus, J. H., \& lyengar, S.S. (2005). Intrinsic an extrinsic motivational orientation in the classroom: age differences and academics correlates. Journal of Educational Psychology, 97(2), 184-196.

Martinelli, S. C. \& Bartholomeu, D. (2007). Escala de motivação acadêmica: uma medida de motivação extrínseca e intrínseca. Avaliação Psicológica, 6 (1), 21-31.

Martinelli, S. C. \& Genari, C. H. M. (2009). Relação entre desempenho escolar e orientações motivacionais. Estudos de Psicologia, 14(1), 13-21.

Martinelli, S. C. \& Sisto, F. F. (2010). Motivação de estudantes: um estudo com crianças do ensino fundamental. Avaliação Psicológica, 9(3), 413-420.

Neves, E. R. C. \& Boruchovitch, E. (2007). Escala de avaliação da motivação para aprender de alunos do ensino fundamental (EMA). Psicologia: Reflexão e Crítica,20 (3), 406-413.

Paiva, M. L. M. F. \& Boruchovitch, E. (2010). Orientações motivacionais, crenças educacionais e desempenho escolar de estudantes do ensino fundamental. Psicologia em Estudo, 15(2), 381-389.

Parish, L.E. \& Treasure, D. C. (2003). Physical activity and situational motivation in physical education: influence of the motivation climate and perceived ability. Research Quarterly for Exercise and Sport, 74(2), 173-183.

Prati, L. E. \& Eizirik, M. F. (2006). Da diversidade na passagem para a quinta série do ensino fundamental. Estudos de Psicologia. 23 (3), 289-298.

Ryan, R. M. \& Deci, E. L. (2000). Self-determination theory and the facilitation of intrinsic motivation, social development, and wellbeing. American Psychologist, 55(1), 68-78.

Thomas, J. \& Nelson, J. (2002). Métodos de pesquisa em atividade física. Porto Alegre: Artmed.

Valentini, N. C. (2002). Percepção de competência e desenvolvimento motor de meninos e meninas: um estudo transversal. Revista Movimento, 8 (2), 51-62.

Valentini, N. C. (2006). Competência e autonomia: desafios para a educação física escolar. Revista Brasileira de Educação Física e Esporte, 20 (5), 85-87.

Villwock, G. (2005). O estudo desenvolvimentista da percepção de competência atlética, da orientação motivacional, da competência motora e suas relações em crianças de escolas públicas. Dissertação de Mestrado,Universidade Federal do Rio Grande do Sul, Porto Alegre-RS.

Villwock, G. \& Valentini, N. C. (2007). Percepção de competência atlética, orientação motivacional e competência motora em 
crianças de escolas públicas: estudo desenvolvimentista e correlacional. Revista Brasileira de Educação Física e Esporte, 21(4), 245-57.

Weiss, M. R. \& Amorose, A. J. (2005). Children's self-perceptions in the physical domain: between - and within - age variability in level, accuracy, and sources of perceived competence. Journal of Sport e Exercise, 27 (2), 226-244.

White, R. W. (1959). Motivation reconsidered: the concept of competence. Psychological Review, 66(5), 297-333.
Wigfield, A. \& Guthrie, J. T. (1997). Motivation for reading: in overview. Educational Psychologist, 32(2), 57-58.

Zisimopoulos, D. A. \& Galanaki, E. P. (2009). Academic intrinsic motivation and perceived academic competence in greek elementary students with and without learning disabilities. Learning Disabilities Research e Practice, 24(1), 33-43.

\section{Sobre as autoras}

Simone Maria Pansera (monipansera@hotmail.com)

Professora do Município de Cachoeirinha-RS, mestre em Ciências do Movimento Humano, Universidade Federal do Rio Grande do Sul.

Nadia Cristina Valentini (nadiacv@esef.ufrgs.br)

Professora do Departamento de Educação Física da Universidade Federal do Rio Grande do Sul, doutora.

Mariele Santayana de Souza (marielesantayana@gmail.com)

Mestranda do Programa de Pós-Graduação em Ciências do Movimento Humano, Universidade Federal do Rio Grande do Sul.

Adriana Berleze (adrianaberleze@yahoo.com.br)

Professora do Departamento de Educação Física da Universidade Federal do Rio Grande do Sul, doutora.

Este estudo é derivado da dissertação de mestrado de Simone Maria Pansera, intitulada "Crianças que se percebem competentes e são intrinsecamente motivadas são motoramente mais competentes? Um estudo associativo entre desenvolvimento motor e aspectos psicossociais de escolares" apresentada ao Programa de Pós-Graduação em Ciências do Movimento Humano da Universidade Federal do Rio Grande do Sul. O estudo teve auxilio do CNPq e CAPES por meio da concessão de bolsas. 